\title{
Organization in short-term recognition memory
}

\author{
P. D. McCORMACK, N. L. CARBONI, and S. P. COLLETTA \\ Carleton University, Ottawa, Ontario, Canada K1S 5B6
}

\begin{abstract}
Two studies were conducted with the purpose of investigating the possibility of organization of information in short-term recognition memory. In the first of these, eight subjects were given extensive testing in a Sternberg setting involving target sets which were either homogeneous (HM) or heterogeneous (HT) with respect to taxonomic category. The functions relating reaction time to set size were parallel for the two treatments and, thus, no evidence was revealed for organization. In the second investigation, 72 subjects were tested once each with set size held constant. Serial position functions for the HT data were consistent with a directed entry strategy and hence with organization in STM. The search for targets in early serial positions appeared to be serial and self terminating, while that for late position items was likely parallel.
\end{abstract}

Two studies, each employing the Sternberg paradigm, (for example; Sternberg, 1969) are of direct relevance to the topic of organization in short-term memory. In one by Naus, Glucksberg and Ornstein (1972), set sizes were $2,4,6$, and 8 and the items were names of animals and girls. In one condition, the set was homogeneous (HM) with respect to taxonomic category, while in the other, it was heterogeneous (HT); that is, girls's or animal's names, but not both, were employed under Condition HM, while an equal number of each, blocked by category, made up Condition HT. The probe followed the termination of the last target by $2 \mathrm{sec}$ on all trials, with subjects being instructed to try to make use of the categorized information. Naus, et al. (1972) considered two possible search strategies for Condition HT, directed entry and random entry. With a directed entry strategy, the subject would presumably perform a serial exhaustive search of the relevant category only, resulting in a $50 \%$ reduction, with respect to Condition HM, in the slope of the function relating RT to set size. With a random entry strategy, on the other hand, the subject would be expected to terminate his search at the category boundary on half of the trials on the average; that is, on those trials where the category of the probe and that of the items of the target set are congruent. The remaining trials would require a serial exhaustive search of all targets. This strategy would result in a $25 \%$ reduction in slope. Their data were consistent with the random-entry strategy.

The second investigation is one by Kaminsky and DeRosa (1972). Their subjects encountered six targets on all trials, some of these being letters and other digits. The subset sizes were either 2,3 , or 4 . An informative cue, indicating whether the search should be for letters

Support for this research was received under National Research Council of Canada Grant APA-78. The authors are indebted to Lydia Little for computer programming. The paper was read at the 1974 meetings of the Psychonomic Society. Requests for reprints should be sent to P. D. McCormack, Department of Psychology, Carleton University, Ottawa, Ontario, Canada, K1S 5 B6. or for digits, was introduced $.4 \mathrm{sec}$ following the termination of the last target and remained visible for $.4 \mathrm{sec}$. The probe was presented $2.0 \mathrm{sec}$ later. The function relating $\mathrm{RT}$ to subset size was increasing and linear, which, in conjunction with other findings, led these investigators to conclude that the subject directed his search to the relevant category, searched that category serially and exhaustively, and then terminated the search at the category boundary. It appears, therefore, that the availability of an informative cue results in a greater amount of organization than that observed where the subject is left on his own, as was the case in the Naus et al. (1972) investigation.

The first experiment to be reported here was similar in design to the Naus et al. (1972) study but it incorporated the informative cue procedure of Kaminsky and DeRosa (1972). It represents one of a series of studies conducted in our laboratories over the past 3 years where, in every case, the results were negative; that is, identical slopes were obtained for conditions HM and HT. Our subjects, therefore, appear to be engaged in a serial, exhaustive search of the total target set even when the materials are categorized and when an informative cue is available. There were, however, some serial position (SP) data of interest in these studies which prompted us to conduct the second investigation to be reported here. Serial position functions were not reported in either the Naus et al. (1972) or the Kaminsky and DeRosa (1972) study. In the second investigation, a procedure much like that of Kaminsky and DeRosa (1972) was employed, with a fixed set size of six and an equal number of items in each of two categories for Condition HT. As will be seen, the data indicate that the search is directed to the relevant category, and if the targets of the category occupy SPs 1-3, the search appears to be serial and self-terminating, whereas the items in SPs 4-6 are likely processed in parallel. 


\section{EXPERIMENT I}

\section{Method}

Subjects. Four male and four female undergraduate students were paid $\$ 10$ each for six 1-h sessions. Each session involved 240 trials. Two males and two females were required to recall the targets during the 5 -sec intertrial interval (Condition FR) with the other four subjects not required to do so (Condition $\overline{F R})$. One male and one female subject in each of these two conditions activated the "yes" button with the preferred hand and the "no" button with the nonpreferred hand. This procedure was reversed for the remaning two subjects.

Materials. Twelve items were selected from each of two taxonomic categories, four-footed animals and girl's first names (Battig \& Montague, 1969). These were the categories employed by Naus et al. (1972). Each of the 24 items was from 3-5 letters long and contained no more than two syllables. There were no obvious high associates among them and they were selected to be as phonemically dissimilar as possible.

Experimental Design. Each subject participated in one practice and five experimental sessions. In each session there were five blocks of 48 trials with a 2-min rest between blocks. The number of items in the positive set was either 2,4 , or 6 . There were two types of sets, one in which the targets were homogeneous with respect to category (Condition HM) and one where they were heterogenous (Condition HT). In the latter condition, the items were blocked by category, with an equal number from each, the animal names appearing in the first part of the list on half the trials and in the last part on the remaining trials. Half the probes were positive and half negative. For Condition HM, the probe was always from the relevant category and for Condition HT, each of the two categories was probed equally often. In every block of 48 trials, there were four trials involving each of the 12 subtreatments resulting from the combination of the three set sizes, the two category conditions, and the two types of probes; otherwise, the selection of a particular subtreatment, as well as the individual items, for any given trial was made at random.

Apparatus and procedure. All probes were presented by means of $35-\mathrm{mm}$ slides mounted in trays in a projector which was located behind an opaque, white $28 \times 23 \mathrm{~cm}$ screen at a distance of $1.4 \mathrm{~m}$. The subject was located $46 \mathrm{~cm}$ in front of the screen and viewed the probe items at eye level. A movable $14 \times 10 \mathrm{~cm}$ response unit was positioned horizontally on a desk between the subject and the screen. On this unit were mounted two pushbuttons, $5.7 \mathrm{~cm}$ apart, one button labeled "yes" and the other labeled "no." The experimenter was located between the subject and the projector and normally out of his visual field. The experimenter's panel consisted of a latency-recording device, two lights in series with the response buttons, a switch for slide projection, a buzzer, a microphone and a metronome.

On each trial, the buzzer was followed immediately by the target set. The targets were presented orally by means of the microphone-speaker system with approximately $1 \mathrm{sec}$ between each target item. Following the last target, the buzzer sounded once more and then the informative cue, "girl" or "animal," was delivered orally. The probe was then presented by means of the projector. The subject's response stopped the timer which was initiated by the onset of the probe. The experimenter then recorded the subject's RT, as well as which button he had pressed. The timing sequence for each trial, which was paced by the metronome, resulted in a 2-sec interval between the last target and the probe. A similar interval was employed by Naus et al. (1972). The interval between the informative cue and the probe was approximately $.5 \mathrm{sec}$ and therefore was considerably shorter than the 2.4-sec interval of the Kaminsky and DeRosa (1972) study. A shorter interval was selected in order to minimize the likelihood that the subject would "dump" half the set under Condition HT. The free recall requirement for half of the subjects was introduced with the same objective in mind.

\section{Results}

The RT data for each subject were subjected to analysis of variance with $\mathrm{A}$ equal to set size $(2,4,6)$, B equal to category condition (HM and HT), ( equal to session (2-6), D equal to probe (positive and negative). Although there were very few inordinately long-latency responses, an alpha of .01 was selected in order to guard against the possibility of committing Type 1 errors. The main effect of A was significant for all eight subjects, RT increasing linearly with set size. The main effect of B was also statistically reliable for all subjects, RTs being longer for Condition HM than for Condition HT. Every subject also exhibited a significant $C$ effect, RTs becoming shorter from Sessions 2-6. For seven of the eight subjects, the main effect of D was statistically dependable, RTs being longer for negative than for positive probes. There were few statistically reliable interactions, and those that were never involved more than two of the eight subjects. The interaction of most relevance to the organization issue, the $A B$ interaction, was significant for only one subject. This particular subject had been assigned to Condition $\overline{\mathrm{FR}}$, and the slope of his function relating RT to set size was less steep under Condition HT than under Condition HM. The amount of slope reduction was closer to $25 \%$ than to $50 \%$ and, therefore, his data were more consistent with a random than with a directed entry strategy. All remaining subjects, however, exhibited parallel functions.

Error rates ranged from $.9 \%$ to $4.5 \%$. Free recall, when required, was $95 \%$ accurate with more omissions occurring under Condition HM than under Condition HT. Although the RT data failed to provide evidence for organization, the subjects typically reported having employed a directed entry strategy for the categorized lists. The SP data for Set Size 6 offered some confirmation of this. However, since each position was not probed equally often, these data may only be considered as being suggestive, and so they will not be reported here. These observations, however, provided the rationale for Experiment II.

\section{EXPERIMENT II}

\section{Method}

Subjects. The subjects were 36 male and 36 female undergraduate students, the majority of them being enrolled in an introductory psychology course. Each subject participated in a single session lasing about $3 / 4 \mathrm{~h}$ and involving 48 trials. Eighteen males and 18 females were required to recall the targets during the intertrial interval (Condition FR), the remaining 36 subjects were not required to do so (Condition $\overline{\mathrm{FR}}$ ). Within each of the four subtreatments created by the FR- $\overline{F R}$ and male-female splits, 9 subjects activated the "yes" button with the preferred hand and the "no" button with the nonpreferred hand, with the procedure being reversed for the remaining 9 subjects. An attempt was made, when assigning subjects to the eight subtreatments, to have the number of subjects per subtreatment equal after every 8 subjects.

Experimental design and procedure. The materials were those 
of Experiment I. A single session employing a relatively large number of subjects was chosen with the purpose of determining whether relatively unpracticed subjects would employ organization strategies. The set size was held constant in this experiment, the subject encountering six targets on all 48 trials. The subjects had 24 trials with each of Conditions HM and HT. For Condition HM, 12 trials involved girl's names and 12 animals's names. The actual names for any given trial were randomly determined. All HM probes were members of the positive set category. For Condition HT, 12 trials had three girl's names followed by three animal's names, while the category order was reversed for the other 12 trials. Again, the actual names for any given trial were randomly determined. In each of the 12-trial segments referred to above, there were six positive and six negative probes. Each of the serial positions was probed once. The 48 trials, therefore, consisted of 24 positive and 24 negative probes, each serial position being probed twice under each of Conditions HM and HT. The mean of these two observations was used for purposes of statistical analysis. Whenever negative-probe data were analyzed, the median of the 12 observations for each of Conditions HM and HT served as the statistical unit. Given the above constraints, the nature of the particular subtreatment was randomly determined for each of the 48 trials.

There were minor procedural changes from Experiment $I$. Because the subjects were relatively unpracticed the intertrial interval was extended from 5 to $10 \mathrm{sec}$ in order to allow those subjects in Condition FR adequate time for recall. The buzzer was replaced by a bell. The microphone-speaker system was disconnected, since it was deemed unnecessary, and the experimenter paced himself by means of tones, recorded at the appropriate intervals, heard through earphones. A 48-trial practice session, with the letters A-L and the digits 1-9, preceeded the experimental session.

\section{Results}

Since the major variable of interest was SP, an analysis of variance was performed only on those data generated under the positive-probe condition. The between-subjects comparison, FR vs. $\overline{\mathrm{FR}}$, which could not be properly evaluated in Experiment I, revealed longer RTs for Condition FR than for Condition $\overline{\mathrm{FR}}$, although the effect did not reach an acceptable level of significance, $F(1,70)=3.53, .05<\mathrm{p}<.10$. There were two within subjects comparisons, SP (1-6) and Category Condition (HM vs. HT). In both cases, there were reliable differences among the means; for $\mathrm{SP}, \mathrm{F}(5,770)=$ $4.99, \mathrm{p}<.001$, and for category condition, $\mathrm{F}(1,770)=$ $52.78, \mathrm{p}<.001$. There was, however, a significant second-order interaction, $F(5,770)=2.43, p<.05$, which mainly reflected the fact that the nature of SP by Category Condition interaction was different under Condition FR than under Condition $\overline{\mathrm{FR}}$. For this reason, it was decided to perform four separate SP analyses, the $\mathrm{F}$ equals ratios $(5,175)$ being as follows: FR-HM, 2.34 $(\mathrm{p}<.02)$; FR-HT, 1; FR-HM, 3.51, $(\mathrm{p}<.01)$; $\overline{\text { FR }}-\mathrm{HT}$, $3.45(\mathrm{p}<.01)$. These data are summarized in Figure 1 where RT is shown as a function of SP for each of the four conditions. Setting alpha at .01 , critical differences were established for the means for each of the three conditions for which significant SP effects were revealed. For the FR-HM treatment, the critical difference was $155.18 \mathrm{msec}$ and therefore there were statistically

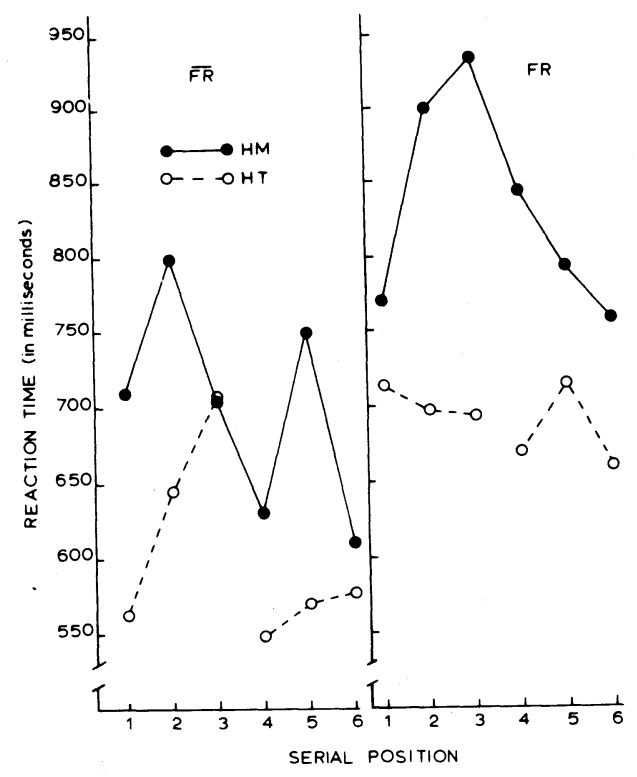

Figure 1. RT as a function of serial position for Conditions FR-HM, FR-HT, FR-HM and FR-HT (Experiment II).

reliable differences between SPs 1 and 3 and 3 and 6 . For Condition $\overline{\mathrm{FR}}-\mathrm{HM}$, the critical difference was $124.72 \mathrm{msec}$ resulting in significant differences between SPs 2 and 4, 2 and 6, and 5 and 6. Finally, the critical difference for Condition FR-HT was $109.13 \mathrm{msec}$, the following means being reliably different: 1 and 3,3 and 4, 3 and 5 , and 3 and 6.

In order to test for differences between the positive and negative probe conditions, the data were collapsed for SP, resulting in one between-subjects effect (FR vs. $\overline{\mathrm{FR}}$ ) and two within-subjects effects (HM vs. HT, and positive vs. negative probes). The analysis of variance revealed that RTs were slower with negative than with positive probes, $\mathrm{F}=11.66(1,210), \mathrm{p}<.001$. There were no reliable interactions involving the probe condition. The average error rate was $2.3 \%$ and free recall was $88 \%$ accurate. Recall was slightly poorer than that reported in Experiment I, but this is not surprising in the light of the fact that the subjects of that study were considerably more practiced.

\section{DISCUSSION}

The parallel HM and HT functions of Experiment I represent yet another failure on our part to replicate the findings of Naus et al. (1972), whose data were consistent with a random entry strategy. This failure occurred in spite of the use of an informative cue like that employed by Kaminsky and DeRosa (1972). 'It will be recalled that these latter investigators obtained evidence for directed search. Their informative cue condition, however, may have been more effective than ours because of the longer cue-probe interval which might have enabled the subject to "dump" the irrelevant subset. At least on the surface, the data of Experiment I appear to indicate that, even under 
Condition HT, the subject will search the total target set and, therefore, fail to take advantage of the category information. The search appears to be of the standard serial exhaustive variety since RT was a linear function of set size and the positive and negative probe slopes were parallel.

The SP data of both experiments, on the other hand, especially those of Experiment II, lead to quite different conclusions regarding organization in STM. It is clear from Figure 1 that, under Condition $\overline{\mathrm{FR}}-\mathrm{HT}$, the subjects directed their search to the cued category. This may be inferred from the absence of a significant difference between SPs 1 and 4, those SPs having the shortest RTs. The systematic increase in RT over SPs 1-3 appears to indicate that the search process for these targets was of the serial. self-terminating variety. A flat function would be expected should the search be serial and exhaustive. For targets occupying SPs 4-6, on the other hand, a parallel search seems to have occurred. A serial strategy seems unlikely in the light of the large differences in RT between SPs 3 and 4, 3 and 5 , and 3 and 6 . A parallel search would appear to be the most efficient for these most recently encountered targets, given the assumption that "echoic memory" is somehow involved (see Neisser, 1967), an assumption which is reasonable in the light of the fact that the interval between the last target and the probe was brief and the targets were presented auditorially (see Kirsner \& (raik, 1971).

The RT data of Condition FR-HT, were there were no reliable differences among the six SP means, fail to support a directed entry notion. It appears that the free recall requirement is such that the subject is unable to optimize his recall and to employ a directed-entry strategy simultaneously. Free recall also generates longer latencies, although the difference between Conditions F R and $\overline{F R}$ failed to reach an acceptable level of significance. These FR-HT data are, of course, not inconsistent with a serial exhaustive search notion, but this is unlikely in the light of the data ior the two HM conditions where there were decided SP effects. The data of Condition FR-HM are characterized by both primacy and recency, whereas those of Condition FR-HM appear to be even more complex.

Finally, RTs were significantly longer for Condition HM than for Condition HT, in both experiments. This alone would appear to indicate that organizational strategies might have been employed under Condition HT, possibly even when the subject had to recall the targets, and that these strategies, in turn, speed up the information processing and decision making that such a task demands. This demonstration that information can be organized in STM makes the notion of a uniprocess memory system even more credible (see Craik \& Lockhart, 1972; Melton, 1963).

\section{REFERENCES}

Battig, W. F., \& Montague, W. E. Category norms for verbal items in 56 categories: A replicaton and extension of the Connecticut category norms. Journal of Experimental Psychology Monograph, 1969, 80, No. 3, Part 2.

Craik, F. I. M., \& Lockhart, R. S. Levels of processing: A framework for memory research. Journal of Verbal Learning and Verbal Behvior, 1972, 11,671-684.

Kaminsky, C. A. \& DeRosa, D. V. Influence of retrieval cues and set-organization in short-term recognition memory. Journal of Experimental Psychology, 1972, 96, 449-454.

Kirsner, K., \& Craik, F. I. M. Naming and decision processes in short-term recognition memory. Journal of Experimental Psychology, 1971, 88, 149-157.

Melton, A. W. Implications of short-term memory for a general theory of memory. Journal of Verbal Learning and Verbal Behavior, 1963, 2, 1-21.

Naus, M. Z., Glucksberg, S., \& Ornstein, P. A. Taxonomic word categories and memory search. Cognitive Psychology, 1972, 3, 643-654.

Neisser, U. Cognitive psychology. New York Appleton-Century-Crofts, 1967, Pp. 199-206.

Sternberg, S. Memory scanning: Mental processes revealed by reaction-time experiments. American Scientist, 1969, 57, 421-457.

(Received for publication December 26, 1974.) 